\title{
Natural products and adverse drug interactions
}

$T$ he public generally considers natural products such as "health foods" and herbal remedies to be safe and beneficial. That these substances are largely unregulated contributes to the misconception that they are innocuous: patients don't feel the need to tell their physicians that they are using them, and physicians don't routinely ask patients if they are taking them. Yet there is a potential for these products to alter the action of certain drugs, sometimes with adverse clinical effects.

Natural products can "interact" with drugs by affecting the biological processes that regulate their metabolism and elimination. The family of enzymes $\infty$ known as the cytochrome P450s (CYPs) ${ }^{1}$ are heavily involved in the business of drug metabolism. Of its many subtypes, CYP3A4 is one of the most important; we will use it to illustrate how interactions between natural products and medications can occur.

CYP3A4 has been estimated to at last partly oxidize $60 \%$ of all drugs to metabolites that are either less active or more readily excreted (or both). Its availability in the small intestine and liver enables it to inactivate orally administered drugs before they enter the systemic circulation (first-pass elimination), thereby reducing the drug's bioavailability (the percentage of an oral dose that reaches the systemic circulation unchanged). If the activity of CYP3A4 is altered, so too is the bioavailability of drugs that are oxidized by this enzyme (see Fig. 1).

One way that a natural product can alter the action of an enzyme is to inhibit it. Our group made the initial finding that grapefruit can markedly reduce enteric CYP3A4 activity, thus elevating the bioavailability of drugs metabolized by enteric CYP3A4 to potentially toxic levels. ${ }^{2}$ Grapefruit juice-drug in- teractions have been succinctly discussed in a recent article in this journal. ${ }^{3}$

St. John's wort, a herb that has become popular in the treatment of depression, has the opposite effect. It increases the activity of CYP3A4, thereby causing more complete oxidation of drugs; higher metabolism means lower bioavailability and hence diminished clinical response. Moreover, St. John's wort increases the activity of the drug transporter P-glycoprotein. Like CYP3A4, this transporter is expressed in apical enterocytes and hepatocytes. $\mathrm{P}$-glycoprotein acts to pump drugs back into the intestinal lumen directly from the gut wall and indirectly from the liver via bile, thus reducing the bioavailability of orally administered drugs. ${ }^{4} \mathrm{P}$-glycoprotein is also located in the renal tubules, where it facilitates systemic drug elimination. The combined action of CYP3A4 and P-

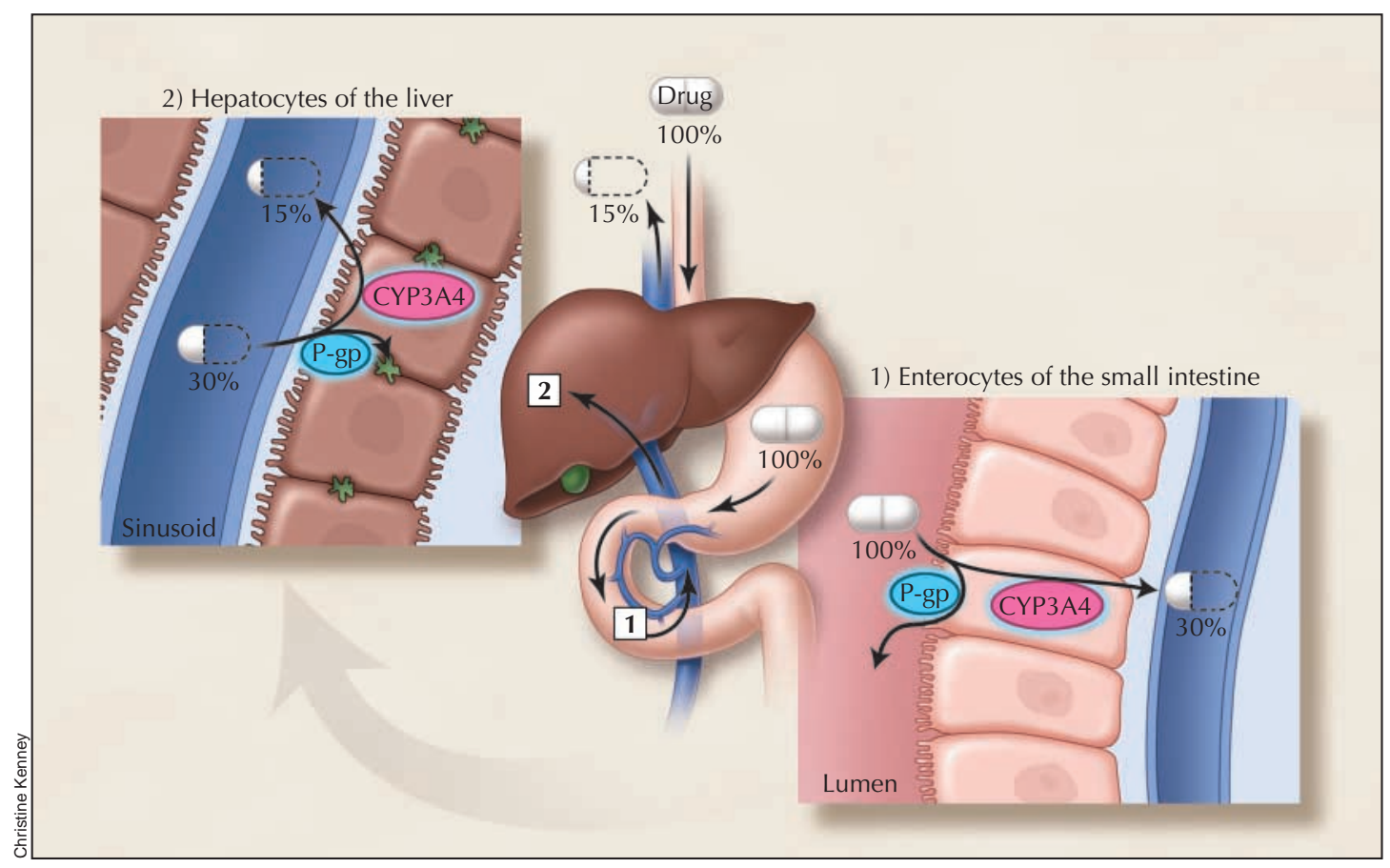

Fig. 1: Sequential first-pass elimination of a theoretical drug through metabolism by CYP3A4 and/or transport by P-glycoprotein (P-gp) in enterocytes of the small intestine and then hepatocytes of the liver. The percentage of the initial drug dose that is available before and after passage through the gut wall and liver is presented. Although the drug is $100 \%$ absorbed from the gastrointestinal tract, its bioavailability is only $15 \%$ after oral administration. 
glycoprotein in the gut and liver can substantially decrease bioavailability and diminish effectiveness. The mechanism by which St. John's wort enhances the activities of CYP3A4 and Pglycoprotein is interesting. It does not involve an actual increase in the activity of individual enzyme and transporter molecules but, rather, induces transcription and translation, resulting in a higher quantity of CYP3A4 and P-glycoprotein. This effect is usually evident within a few days of repeated administration and lasts for approximately a week after discontinuation of St. John's wort.

The clinical consequences of diminished drug efficacy can be as devastating as those of enhanced drug toxicity. For example, St. John's wort was been shown to decrease plasma concentrations of cyclosporine and indinivir sufficiently to cause, respectively, organ rejection and increased HIV viral load. ${ }^{5,6} \mathrm{Al}$ though plasma concentrations of cyclosporine are routinely monitored, the basis for an observed loss of benefit during indinivir therapy may go unrecognized, possibly leading to the inappropriate discontinuation of an effective medication or the unnecessary addition of other drugs. Moreover, a broad range of medications appears to be affected. The list includes highly prescribed medications that are substrates for CYP3A4 $4^{3}$ or $\mathrm{P}$ glycoprotein (digoxin, HIV protease inhibitors) and others that have been implicated in case reports (amitriptyline, theophylline, ethinylestradiol + desogestrel, warfarin).

Physicians should be wary that abrupt and unexplained changes or variability in the clinical status of a patient may be the result of a natural product-drug interaction; it is easy for substances such as grapefruit or herbal remedies to be overlooked as an underlying cause. Also, most herbal products have not been assessed for their potential to cause drug interactions. As a general rule, we recommend discouraging the use of those products for which there is no information. However, many patients will continue to use grapefruit and herbal medications regardless. Rather than immediately altering medication regimens in response to a change in a patient's clinical condition, taking a careful dietary and herbal history may be a more effective initial approach. Although the Compendium of Pharmaceuticals and Specialties (Clin-Info Section, Product Monographs) may be the most popular source of drug information among clinicians, software programs (e.g., Epocrates) and Internet sites (e.g., www.drug-interactions .com) can also be useful.

David G. Bailey

Lawson Health Research

Institute, London Health

Sciences Centre

Departments of Medicine and

Physiology \& Pharmacology

George K. Dresser

Lawson Health Research

Institute, London Health

Sciences Centre

Department of Medicine

University of Western Ontario

London, Ont.

\section{References}

1. Dresser GK, Spence JD, Bailey DG. Pharmacokinetic-pharmacodynamic consequences and clinical relevance of cytochrome P450 3A4 inhibition [review]. Clin Pharmacokinet 2000;38:41-57.

2. Bailey DG, Spence JD, Munoz C, Arnold JMO. Interaction of citrus juices with felodipine and nifedipine. Lancet 1991;337:268-9.

3. Maskalyk J. Grapefruit juice: potential drug interactions. CMAF 2002; 167:279-80.

4. Lin JH, Yamazaki M. Role of Pglycoprotein in pharmacokinetics: clinical implications [review]. Clin Pharmacokinet 2003;42:59-98.

5. Ruschitzka F, Meier PJ, Turina M, Luscher TT, Noll G. Acute heart transplant rejection due to Saint John's wort. Lancet 2000;355:548-9.

6. Piscitelli SC, Burstein AH, Chaitt D, Alfaro RM, Falloon J. Indinavir concentrations and St John's wort [published erratum appears in Lancet 2001;357:1210]. Lancet 2000;355:547-8. 